\title{
INTEGRAL OPERATORS AND UNIVALENT FUNCTIONS
}

\author{
KIAH WAH ONG, SIN LENG TAN AND YONG ENG TU
}

\begin{abstract}
In this paper, we define two new integral operators $L^{k}$ and $L_{k}$ which are iterative in nature. We show that for $f(z)=z+a_{2} z^{2}+\cdots+a_{n} z^{n}+\cdots$ with radius of convergence larger than one, $L^{k} f(z)$ and $L_{k} f(z)$ when restricted on $E=\{z:|z|<1\}$ will eventually be univalent for large enough $k$. We then show that these are the best possible results by demonstrating that there exists a holomorphic function $T(z)$ in normalized form and with radius of convergence equal to one such that $L^{k} T(z)$ and $L_{k} T(z)$ fail to be univalent when restricted to $E$ for every $k \in \mathbb{N}$.
\end{abstract}

\section{Introduction}

Let $E$ be the unit disk $\{z:|z|<1\}$ and $S$ be the set of univalent functions in $E$ which can be normalized to the conditions $f(0)=0$ and $f^{\prime}(0)=1$. In 1960 Biernacki [1] falsely claimed that $F(z)=\int_{0}^{z} \frac{f(\zeta)}{\zeta} d \zeta$ is in $S$ whenever $f \in S$. Three years later this error was notice by Krzyz and Lewandowski [3, p.149]. Nevertheless, Biernacki's consideration of the integral transform gave rise to the study of the following problem:

for what choices of the parameter $\alpha$ is $F_{\alpha}(z)=\int_{0}^{z}\left(\frac{f(\zeta)}{\zeta}\right)^{\alpha} d \zeta$ in $S$ whenever $f \in S$.

$F_{\alpha}(z)$ defined above is known as integrals of the first type. Many results were obtained by Merkes and Wright [4] in the study of this integral. Other types of integral operators are also studied by researchers in this area. For example $G_{\alpha}(z)=\int_{0}^{z}\left[f^{\prime}(\zeta)\right]^{\alpha} d \zeta$ is known as integrals of the second type and theorems similar to $F_{\alpha}(z)$ were proved by Pfaltzgraff [5], Merkes and Wright [4]. What we consider in this paper are slightly different, we are interested in iterative integral operators that take normalized functions which are not necessarily univalent into the class $S$ when restricted to $E$.

Corresponding author: Kiah Wah Ong.

2010 Mathematics Subject Classification. Primary 30C55; secondary 30C45.

Key words and phrases. Univalent functions, integral operator. 


\section{Integral Operators $L^{k}$ and $L_{k}$}

We define $S_{R \geq 1}$ as the class of holomorphic functions $f$ which are normalized and have radius of convergence $R$, where $R$ is larger or equal to one. Thus for each $f \in S_{R \geq 1}, f$ has a Taylor series expansion of the form

$$
f(z)=z+a_{2} z^{2}+a_{3} z^{3}+\cdots, \quad|z|<R, \text { for } R \geq 1 .
$$

We define $S_{R>1}$ as the class of holomorphic functions $f$ which are normalized and have radius of convergence $R$, where $R$ is strictly greater than one.

For $f \in S_{R \geq 1}$ or $f \in S_{R>1}, f$ has a Taylor series expression of the form $f(z)=z+a_{2} z^{2}+\cdots$, hence $z=0$ is a removable singularity of $f(z) / z$. If we define $f(z) / z=1$ when $z=0$, then $f(z) / z$ is holomorphic and the complex integral is independent of path.

We then introduce the following two operators which act on $f \in S_{R \geq 1}$ and their definitions are given below.

Definition 2.1. For $f(z) \in S_{R \geq 1}$ let

$$
\begin{aligned}
L f(z) & =\int_{0}^{z} \frac{1}{z_{1}} f\left(z_{1}\right) d z_{1} \\
& =z+\sum_{n=2}^{\infty} \frac{1}{n} a_{n} z^{n} . \\
L^{2} f(z) & =L(L f(z)) \\
& =z+\sum_{n=2}^{\infty} \frac{1}{n^{2}} a_{n} z^{n} .
\end{aligned}
$$

In general, for positive integer $k$, we have

$$
\begin{aligned}
L^{k} f(z) & =L\left(L^{k-1} f(z)\right) \\
& =z+\sum_{n=2}^{\infty} \frac{1}{n^{k}} a_{n} z^{n} .
\end{aligned}
$$

Definition 2.2. For $f \in S_{R \geq 1}$, let

$$
\begin{aligned}
L_{k} f(z) & =\frac{k !}{z^{k-1}} \int_{0}^{z} \int_{0}^{z_{k}} \int_{0}^{z_{k-1}} \cdots \int_{0}^{z_{2}} \frac{1}{z_{1}} f\left(z_{1}\right) d z_{1} d z_{2} d z_{3} \cdots d z_{k} \\
& =z+\sum_{n=2}^{\infty} \frac{1}{\left(\begin{array}{c}
n+k-1 \\
n-1
\end{array}\right)} a_{n} z^{n} .
\end{aligned}
$$

Notice that in the Definition 2.1 and 2.2, $L^{1} f(z)$ and $L_{1} f(z)$ are both equal to $F(z)=$ $\int_{0}^{z} \frac{f(\zeta)}{\zeta} d \zeta$ which is the integral consider by Biernacki in his 1960 paper. We next show that 
for $f \in S_{R>1}$, there exists a positive integer $N$ such that if $k \geq N$, then $L^{k} f(z)$ and $L_{k} f(z)$ restricted to $E$ are univalent and belongs to $S$.

We will need the following results for the proof in the next section. The first is the celebrated Bieberbach's theorem proved by Louis de Branges in 1985. For the proof of this theorem, please refer to [2].

Theorem 2.1 (Bieberbach's Theorem). If

$$
f(z)=z+\sum_{n=2}^{\infty} a_{n} z^{n}
$$

is in $S$, then $\left|a_{n}\right| \leq n$. The inequality is sharp with equality occurs iff $f$ is a rotation of the Koebe function.

Noshiro and Warschawski [6] gave a simple but important criterion for univalence in 1935, this criterion now bears their names.

Theorem 2.2 (Noshiro-Warschawski Theorem). Suppose that $f$ is holomorphic in a convex domain $D$ and for some real $\alpha$ we have

$$
\operatorname{Re}\left\{e^{i \alpha} f^{\prime}(z)\right\}>0
$$

for all $z \in D$. Then $f(z)$ is univalent in $D$.

\section{Main results}

Theorem 3.1. For $f \in S_{R>1}$, there exists a positive integer $N$ such that if $k \geq N$, then $L^{k} f(z)$ restricted to $E$ is univalent and belongs to $S$.

Proof. For fixed $k \in \mathbb{N}$ and $f \in S_{R>1}$. We have

$$
\begin{aligned}
\left\{L^{k} f(z)\right\}^{\prime} & =1+\sum_{n=2}^{\infty} \frac{1}{n^{k-1}} a_{n} z^{n-1} . \\
\operatorname{Re}\left\{L^{k} f(z)\right\}^{\prime} & =1+\sum_{n=2}^{\infty} \frac{1}{n^{k-1}} \operatorname{Re}\left\{a_{n} z^{n-1}\right\} \\
& =1+H(z) .
\end{aligned}
$$

Note that $H(z)=\sum_{n=2}^{\infty} \frac{1}{n^{k-1}} \operatorname{Re}\left\{a_{n} z^{n-1}\right\}$, and

$$
\begin{aligned}
|H(z)| & \leq \sum_{n=2}^{\infty} \frac{1}{n^{k-1}}\left|\operatorname{Re}\left\{a_{n} z^{n-1}\right\}\right| \\
& \leq \sum_{n=2}^{\infty} \frac{1}{n^{k-1}}\left|a_{n} z^{n-1}\right| \\
& \leq \sum_{n=2}^{\infty} \frac{1}{n^{k-1}}\left|a_{n}\right|, \text { for }|z|<1 .
\end{aligned}
$$


Since $f \in S_{R>1}$, then the radius of convergence $R$ for $f$, is greater than 1 . Hence there exists an $\epsilon>0$ such that $B=\frac{1}{R}+\epsilon<1$. From limsup $\left|a_{n}\right|^{\frac{1}{n}}=\frac{1}{R}$ and property of limit supremum, there exists $N_{1} \in \mathbb{N}$ such that if $n \geq N_{1}$, then $\left|a_{n}\right|<B^{n}$.

Let $M=\max \left\{\left|a_{2}\right|,\left|a_{3}\right|, \cdots,\left|a_{N_{1}-1}\right|\right\}$, we have

$$
|H(z)|<\frac{1}{2^{k-1}}\left(N_{1}-2\right) M+\frac{1}{N_{1}^{k-1}}\left(\frac{B^{N_{1}}}{1-B}\right) .
$$

Now, there exists $N \in \mathbb{N}$ such that $|H(z)|<\frac{1}{2}$ whenever $k \geq N$. With this $N \in \mathbb{N}$ we see that

$$
\begin{aligned}
\operatorname{Re}\left\{L^{k} f(z)\right\}^{\prime} & =1+\sum_{n=2}^{\infty} \frac{1}{n^{k-1}} \operatorname{Re}\left\{a_{n} z^{n-1}\right\} \\
& =1+H(z) \\
& >\frac{1}{2}, \text { for }|z|<1
\end{aligned}
$$

whenever $k \geq N$.

By Noshiro-Warschawski theorem, we conclude that $L^{k} f(z)$ is univalent in $E$, whenever $k \geq$ $N$.

We give the following lemma before we prove the next theorem.

Lemma 3.2. For $j, k \in \mathbb{N}$, we have

$$
\frac{j+1}{\left(\begin{array}{c}
j+k \\
j
\end{array}\right)} \leq \frac{j}{\left(\begin{array}{c}
j+k-1 \\
j-1
\end{array}\right)}
$$

Proof. Observe the following trivial inequality $\frac{j+1}{j+k} \leq 1$, multipying both sides of the inequality by $\frac{j !}{(j+k-1) !}$, we obtained $\frac{(j+1) !}{(j+k) !} \leq \frac{j !}{(j+k-1) !}$. The last inequality implies that

$$
\frac{j+1}{\frac{(j+k) !}{k ! j !}} \leq \frac{j}{\frac{(j+k-1) !}{k !(j-1) !}}
$$

This is what we wanted to show.

Theorem 3.3. For $f \in S_{R>1}$, there exists a positive integer $N$ such that if $k \geq N$, then $L_{k} f(z)$ restricted to $E$ is univalent and belongs to $S$.

Proof. For fixed $k \in \mathbb{N}$ and $f \in S_{R>1}$, we have

$$
\begin{aligned}
\left\{L_{k} f(z)\right\}^{\prime} & =1+\sum_{n=2}^{\infty} \frac{n}{\left(\begin{array}{c}
n+k-1 \\
n-1
\end{array}\right)} a_{n} z^{n-1} . \\
\operatorname{Re}\left\{L_{k} f(z)\right\}^{\prime} & =1+\sum_{n=2}^{\infty} \frac{n}{\left(\begin{array}{c}
n+k-1 \\
n-1
\end{array}\right)} \operatorname{Re}\left\{a_{n} z^{n-1}\right\} \\
& =1+G(z) .
\end{aligned}
$$


Note that $G(z)=\sum_{n=2}^{\infty} \frac{n}{\left(\begin{array}{c}n+k-1 \\ n-1\end{array}\right)} \operatorname{Re}\left\{a_{n} z^{n-1}\right\}$, and

$$
\begin{aligned}
|G(z)| & \leq \sum_{n=2}^{\infty} \frac{n}{\left(\begin{array}{c}
n+k-1 \\
n-1
\end{array}\right)}\left|\operatorname{Re}\left\{a_{n} z^{n-1}\right\}\right| \\
& \leq \sum_{n=2}^{\infty} \frac{n}{\left(\begin{array}{c}
n+k-1 \\
n-1
\end{array}\right)}\left|a_{n} z^{n-1}\right| \\
& \leq \sum_{n=2}^{\infty} \frac{n}{\left(\begin{array}{c}
n+k-1 \\
n-1
\end{array}\right)}\left|a_{n}\right|, \text { for }|z|<1 .
\end{aligned}
$$

Since $f \in S_{R>1}$, then the radius of convergence $R$ for $f$, is greater than 1 . Hence there exists an $\epsilon>0$ such that $A=\frac{1}{R}+\epsilon<1$. From limsup $\left|a_{n}\right|^{\frac{1}{n}}=\frac{1}{R}$ and property of limit supremum, there exists $N_{1} \in \mathbb{N}$ such that if $n \geq N_{1}$, then $\left|a_{n}\right|<A^{n}$.

Let $M=\max \left\{\left|a_{2}\right|,\left|a_{3}\right|, \cdots,\left|a_{N_{1}-1}\right|\right\}$, and from Lemma 3.2 we have

$$
|G(z)|<\frac{2}{k+1}\left(N_{1}-2\right) M+\frac{N_{1} ! k !}{\left(N_{1}+k-1\right) !}\left(\frac{A^{N_{1}}}{1-A}\right) .
$$

Now, there exists $N \in \mathbb{N}$ such that $|G(z)|<\frac{1}{2}$ whenever $k \geq N$. With this $N \in \mathbb{N}$ we see that

$$
\begin{aligned}
\operatorname{Re}\left\{L_{k} f(z)\right\}^{\prime} & =1+\sum_{n=2}^{\infty} \frac{n}{\left(\begin{array}{c}
n+k-1 \\
n-1
\end{array}\right)} \operatorname{Re}\left\{a_{n} z^{n-1}\right\} \\
& =1+G(z) \\
& >\frac{1}{2}, \text { for }|z|<1,
\end{aligned}
$$

whenever $k \geq N$.

By Noshiro-Warschawski theorem, we conclude that $L_{k} f(z)$ is univalent in $E$, whenever $k \geq$ N.

Next, we are going to show that Theorem 3.1 and 3.3 are the best possible results. Let

$$
T(z)=z+\sum_{m=2}^{\infty} a_{m} z^{m}, \text { where } a_{m}=\left\{\begin{array}{l}
s^{s}, \text { if } m=2^{s}, s \in \mathbb{N} \\
0, \text { otherwise }
\end{array}\right.
$$

$T(z)$ has radius of convergence one since limsup $\left|a_{m}\right|^{\frac{1}{m}}=1$. Taking $s>2$ and $m=2^{s}$, we have $a_{m}=s^{s}>2^{s}=m$, hence by the contrapositive of Bieberbach theorem [Theorem 2.3], we conclude that $T(z)$ is not univalent in $E$. We then show that $L^{k} T(z)$ and $L_{k} T(z)$ is not univalent in $E$ for every positive integer $k$.

Theorem 3.4. $L^{k} T(z)$ is not univalent in $E$ for every $k \in \mathbb{N}$. 
Proof. For fixed $k \in \mathbb{N}$.

$$
L^{k} T(z)=z+\sum_{m=2}^{\infty} b_{m} z^{m}, \text { where } b_{m}=\left\{\begin{array}{cl}
\frac{s^{s}}{\left(2^{s}\right)^{k}}, & \text { if } m=2^{s}, s \in \mathbb{N} \\
0, & \text { otherwise }
\end{array}\right.
$$

There exists a $s \in \mathbb{N}$ such that $s>2^{k+1}$. Choose $m=2^{s}$, then we have

$$
b_{m}=\frac{s^{s}}{\left(2^{s}\right)^{k}}=\left(\frac{s}{2^{k}}\right)^{s}>2^{s}=m
$$

By the contrapositive of Bieberbach theorem, we concluded that $L^{k} T(z)$ is not univalent in $E$. Since $k \in \mathbb{N}$ arbitrary, this proves the theorem.

Theorem 3.5. $L_{k} T(z)$ is not univalent in $E$ for every $k \in \mathbb{N}$.

Proof. For fixed $k \in \mathbb{N}$.

$$
L_{k} T(z)=z+\sum_{m=2}^{\infty} c_{m} z^{m}, \text { where } c_{m}=\left\{\begin{array}{cl}
\frac{s^{s}}{\left(2^{s+k-1}\right)}, & \text { if } m=2^{s}, s \in \mathbb{N} \\
2^{s}-1 & \text { otherwise }
\end{array}\right.
$$

Note that there is a $s_{1} \in \mathbb{N}$, such that $2^{s_{1}}>k-1$, for the fixed $k$. Because

$$
\lim _{s \rightarrow \infty} \frac{s}{2^{\frac{1}{s}}}=\infty
$$

there is a $s_{2} \in \mathbb{N}$, such that $\frac{s_{2}}{2^{\frac{1}{s_{2}}}}>2^{k+1}$. Let $s=\max \left\{s_{1}, s_{2}\right\}$, then we have the following inequality.

$$
\frac{s^{s}}{\left(\begin{array}{c}
2^{s}+k-1 \\
2^{s}-1
\end{array}\right)}=\frac{s^{s} k !}{\left(2^{s}+k-1\right)\left(2^{s}+k-2\right) \cdots 2^{s}} \geq \frac{s^{s} k !}{\left(2^{s}+k-1\right)^{k}} \geq \frac{k !}{2^{k}} \frac{s^{s}}{2^{s k}} \text {. }
$$

Note that

$$
\frac{k !}{2^{k}}=\frac{k}{2} \frac{k-1}{2} \frac{k-2}{2} \cdots \frac{3}{2} \frac{2}{2} \frac{1}{2} \geq \frac{1}{2}
$$

hence,

$$
\frac{s^{s}}{\left(\begin{array}{c}
2^{s}+k-1 \\
2^{s}-1
\end{array}\right)} \geq \frac{1}{2} \frac{s^{s}}{2^{s k}}
$$

Since $\frac{s}{2^{\frac{1}{s}}}>2^{k+1}$, we have

$$
\frac{s^{s}}{\left(\begin{array}{c}
2^{s}+k-1 \\
2^{s}-1
\end{array}\right)} \geq \frac{1}{2} \frac{s^{s}}{2^{s k}}>2^{s} .
$$

By taking $m=2^{s}$, we have

$$
c_{m}=\frac{s^{s}}{\left(\begin{array}{c}
2^{s}+k-1 \\
2^{s}-1
\end{array}\right)} \geq \frac{1}{2} \frac{s^{s}}{2^{s k}}>2^{s}=m .
$$

By the contrapositive of Bieberbach theorem, we concluded that $L_{k} T(z)$ is not univalent in $E$. Since $k \in \mathbb{N}$ arbitrary, this proves the theorem. 


\section{References}

[1] M. Biernacki, Sur l'intégrale des fonctions univalentes, Bull. Acad. Polon. Sci. 8 (1960), 29-34.

[2] L. de Branges, A proof of the Bieberbach conjecture, Acta Math. 154 (1985), 137-152.

[3] A. W. Goodman, Univalent Functions, Vol II, Mariner Publishing Co., Tampa, Florida, 1983.

[4] E. P. Merkes and D. J. Wright, On the univalence of a certain integral, Proc. Amer. Math. Soc. 27 (1971), 97-100.

[5] J. A. Pfaltzgraff, Univalence of the integral of $f^{\prime}(z)^{\lambda}$, Bull. London Math. Soc. 7 (1975), 254-256.

[6] S. Warschawski, On the higher derivatives at the boundary in conformal mapping, Trans. Amer. Math. Soc. 38 (1935), 310-340.

Faculty of Engineering and Science, Universiti Tunku Abdul Rahman, 53300 Kuala Lumpur, Malaysia.

E-mail: kiahwah@gmail.com

Faculty of Engineering and Science, Universiti Tunku Abdul Rahman, 53300 Kuala Lumpur, Malaysia.

E-mail: tslen@utar.edu.my

YL Design Consultancy Services, 47500 Petaling Jaya, Malaysia.

E-mail: yongengtu@yahoo.com 